We do not think it possible to confirm the association of this syndrome as reported by Wannarachue et al. ${ }^{4}$ with growth deficiency. Although the mother is only $150 \mathrm{~cm}$ (3rd centile), she comes from a short but otherwise healthy family; the daughter's growth pattern, instead, follows the 20th centile and is at the upper limit of her genetic potential. The aetiology of the Rapp-Hodgkin syndrome is unknown. The mother is apparently the first case from unaffected parentage although it was not possible for us to examine any other members of the family. The expression of the syndrome seems to be the same in both sexes. ${ }^{4}$ Our report supports the hypothesis of autosomal dominant transmission, although $\mathrm{X}$-linked inheritance cannot be excluded.

\section{References}

1 Rapp R S, Hodgkin W E. Anhidrotic ectodermal dysplasia. Autosomal dominant inheritance with palate and lip anomalies. J Med Genet 1968; 5: 269-72.

2 Summitt R L, Hiatt R L. Hypohidrotic ectodermal dysplasia with multiple associated anomalies. Birth Defects 1971; 7: No 8, 121-4.

3 Frias J L, Smith D W. Diminished sweat pores in hypohidrotic ectodermal dysplasia: a new method for assessment. J Pediatr 1968; 72: 606-10.

4 Wannarachue N, Hall B D, Smith D W. Letter: Ectodermal dysplasia and multiple defects (Rapp-Hodgkin's type). J Pediatr 1972; 81 : 1217-8.

Correspondence to Dr Barbara Stasiowska-Randone, via Pinasca 16, 10138 Torino, Italy.

Received 28 May 1981

\title{
Infantile aortic aneurysm complicating umbilical arterial catheterisation
}

\author{
A B COLCLOUGH AND A J BARSON
}

Department of Pathology, St Mary's Hospital, Manchester

SUMMARY A true aneurysm of the lower thoracic aorta in a preterm infant dying of septicaemia at $14 \frac{1}{2}$ weeks is described. This is a rare complication of infection associated with umbilical arterial catheterisation and the first case in which Pseudomonas aeruginosa was the likely causative organism.

Mycotic aortic aneurysm in childhood was a wellknown clinical entity in the days when bacterial endocarditis was more common than it is today. ${ }^{1}$ Aortic aneurysms which present within the first 6 months of life are an even rarer phenomenon, but since 1976 five such cases have been reported each bearing a striking similarity to each other. ${ }^{2-5}$ All have occurred in preterm infants who have had umbilical arterial catheterisation complicated by a staphylococcal septicaemia. The present case differs from these in that a pseudomonas septicaemia appears to have produced a true rather than a false aortic aneurysm.

\section{Case report}

A girl was born at 26 weeks' gestation by normal vertex vaginal delivery after rupture of the membranes 2 weeks previously. She cried at birth but required intubation at 4 minutes for respiratory distress. An infection screen showed no evidence of intrauterine infection and prophylactic gentamicin and penicillin were given for 11 days. From day 1 to
26 an umbilical arterial catheter was in situ with the tip checked radiographically to be at the level of the 8th thoracic vertebra. Respiratory distress syndrome on the first day of life was complicated by interstitial emphysema secondary to high artificial ventilatory pressures. Pneumothorax was followed on day 4 by pneumonia, a haemothorax, and disseminated intravascular coagulopathy. Jaundice necessitated an exchange transfusion via an umbilical venous catheter which was in place from day 3 to 8 . On day 5 the infant's general condition had worsened and Pseudomonas sp. and a coagulase-negative Staphylococcus sp. were cultured from the tip of the endotracheal suction catheter but not from blood. These organisms were again found on day 8 on the endotracheal tube and the venous catheter tip respectively. The pneumonia improved by day 9 when ticarcillin was added to the antibiotic regimen. The baby was extubated on day 11 but the pneumonia worsened. Chloramphenicol was given with some success but anaemia, apnoeic attacks, and episodes of bradycardia persisted. By day 21 the baby became septicaemic and was reventilated for a further 6 days. Pseudomonas sp., Enterobacter sp., and Monilia sp. were cultured from the blood on several occasions during the next few days, and Pseudomonas sp. was also found on the umbilical arterial tip and the endotracheal tube. The septicaemia was treated with ticarcillin, gentamicin, and an exchange transfusion. By day 26 there had been some improvement, the infant was extubated and the umbilical arterial 
catheter replaced by a radial arterial line. Oral feeding was started and the baby nursed in air. There was a relapse on day 36 when the abdomen became distended, with blood-stained stools and episodes of bradycardia. However there were no radiological features of necrotising enterocolitis and the abdominal distension settled. The blood cultures were initially sterile but Staphylococcus aureus was isolated both from blood and stool on day 49 after the abdominal distension had recurred together with worsening pneumonia, apnoeic attacks, and bradycardia. The symptoms settled again with cloxacillin but the chest radiograph remained abnormal with features of bronchopulmonary dysplasia and consolidation. The radial arterial line was removed. The baby was now wasted and was chronically anaemic despite blood transfusions. Cytomegalovirus was cultured from the urine. At 9 weeks Pseudomonas sp. was again cultured from blood and ticarcillin was restarted. After some recovery she began to deteriorate again at 12 weeks with pneumonia,

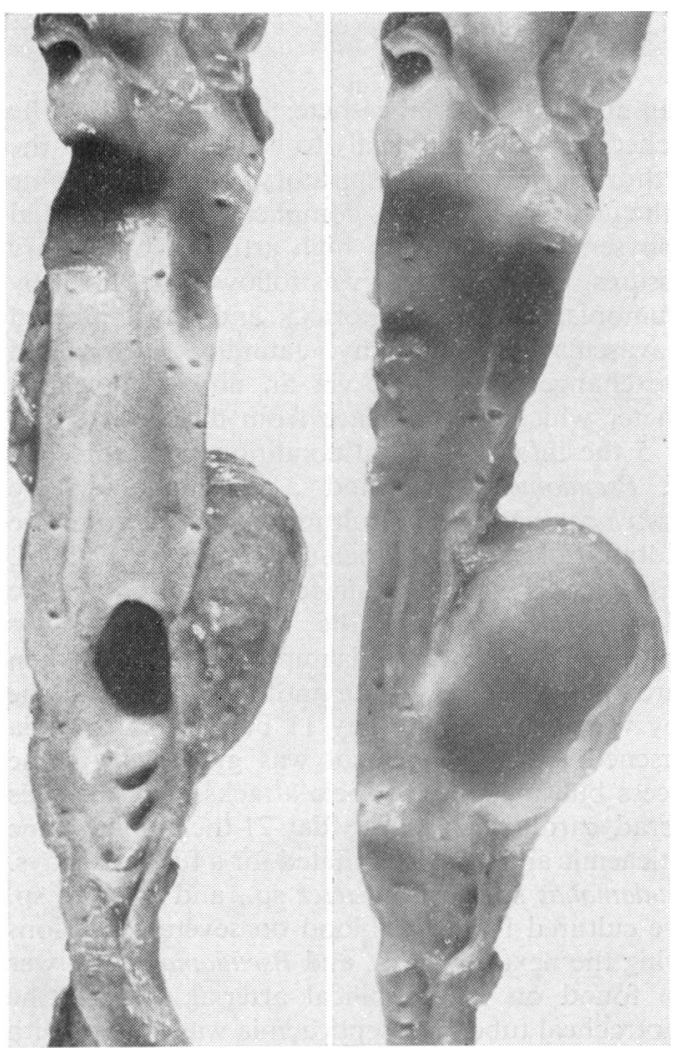

Fig. 1 Thoracic and abdominal aorta opened posteriorly to show the intact aneurysm (left) and its smooth bisected surface (right). $\times 1 \cdot 5$.

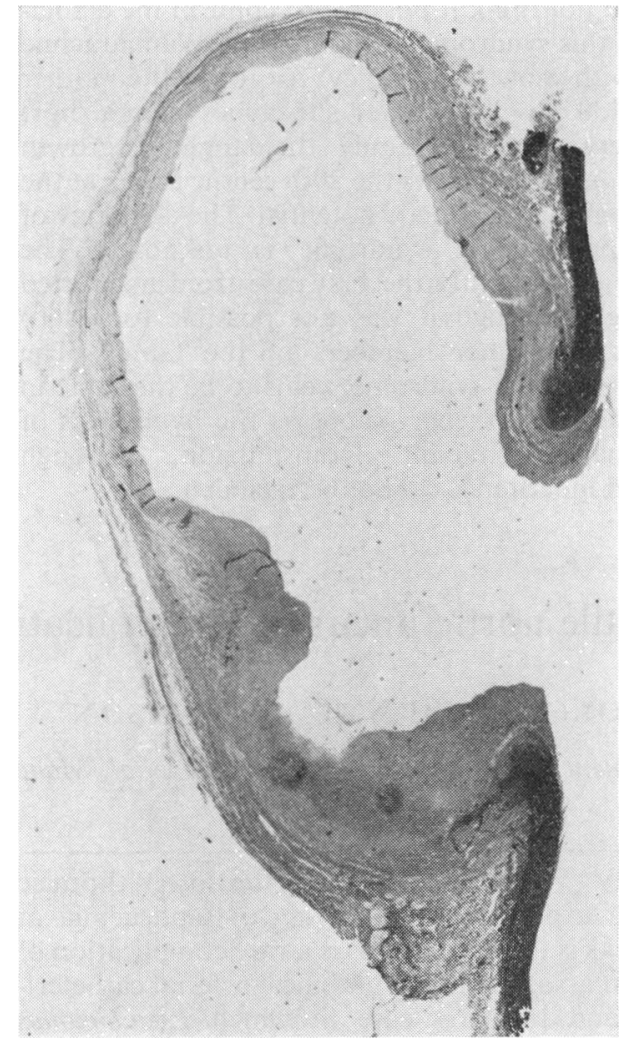

Fig. 2 Sagittal section through the aortic aneurysm showing loss of elastic fibres in the aneurysmal wall. Elastic van Gieson $\times 5$.

pyrexia, ard bradycardia. Despite further chemotherapy there was a sudden collapse at age 14 weeks. The baby was reintubated, ventilated, and a radial arterial line was reinserted. A blood culture grew coagulase-negative Staphylococcus sp. and the endotracheal specimen grew Pseudomonas aeruginosa. There was then progressive deterioration with an unresponsive metabolic acidosis until death at $14 \frac{1}{2}$ weeks.

\section{Necropsy}

A saccular aneurysm of the lower thoracic aorta (Fig. 1) $60 \mathrm{~mm}$ from the aortic valve was found. This measured $19 \times 12 \times 11 \mathrm{~mm}$ with a narrower orifice measuring $8 \times 4 \mathrm{~mm}$. The aneurysm arose from the right anterolateral aspect of the aorta. It had a smooth intimal surface with no adherent thrombus. Microscopical examination showed an abrupt loss of the media and elastic tissue at the orifice of the aneurysm (Fig. 2) leaving only a few strands in the aneurysmal 
wall which otherwise consisted of intima, fibrous connective tissue, and adventitia. Chronic inflammatory cells and haemosiderin containing macrophages were scattered throughout the wall of the aneurysm but appropriate stains for bacteria, including Treponema sp., were negative. A small organising thrombus was seen adherent to the intimal surface of the aorta adjacent to the aneurysm. The aorta elsewhere displayed no evidence of aortitis.

Both lungs showed focal necrotic consolidation and vasculitis typical of pseudomonas bronchopneumonia. There was also a moderate degree of bronchopulmonary dysplasia. Hepatosplenomegaly was noted and ascites. The 12th thoracic vertebra was bifid. Cytomegalovirus inclusions were seen histologically in the kidneys. Cultures of the lungs, blood, and peritoneal fluid at necropsy grew Pseudomonas sp., and cytomegalovirus was isolated in tissue culture from a kidney.

\section{Discussion}

Congenital aneurysm of the aorta is an exceedingly rare entity. The only well documented case is that described by Howorth ${ }^{6}$ in which an abdominal aneurysm measuring $11 \mathrm{~cm}$ in diameter was recognised at birth. The infant did not survive surgery and subsequent microscopical examination showed preservation of all layers of the aorta within the wall of the aneurysm. The replacement of the media and elastin in the wall of the aneurysm in the present case by fibrous tissue in which were chronic inflammatory cells is a strong indication of its mycotic nature even though no bacteria could be demonstrated. Five infants with aortic aneurysm have been described in recent years, ${ }^{2-5} 2$ of whom were twins. ${ }^{5}$ All these infants were known to have had a staphylococcal septicaemia and all had had umbilical arterial catheterisation, 4 as part of the treatment of respiratory distress syndrome and 1 for exchange transfusion. All these infants had a false aneurysm in which rupture of the aortic wall communicated with an aneurysmal sac composed of an organising paraaortic haematoma.

Our case differs from these in that there was a true aneurysm in which the cavity was a distorted, fibrotic, but unruptured aortic wall. Pseudomonas sp., Enterobacter sp., Staphylococcus sp., and Monilia sp. were all cultured from the blood at various times, but Pseudomonas sp. was the most persistent pathogen; it had been isolated from the umbilical arterial tip and was widely diseminated through the tissues at necropsy. Destruction of elastin in the arterial walls with minimal inflammatory response is a characteristic of Pseudomonas sp. infection ${ }^{7}$ and this, rather than Staphylococcus aureus, appears to have been the most likely responsible pathogen.
The variety and persistence of sepsis in this case was in part a reflection of the extreme prematurity of the infant, and the previous cases recorded have all been in preterm infants ranging from 32 to 36 weeks' gestation. Only 2 have had the aneurysm successfully excised. ${ }^{34}$ It has been suggested that local trauma by the catheter is an important factor in the aetiology of the condition ${ }^{2}$ but most authors have felt the aneurysms are essentially mycotic in nature. Moreover there is evidence that aneurysms of the pulmonary artery in infancy may also be caused by Staphylococcus aureus and Streptococcus viridans septicaemia. $^{8}$

The role of the umbilical arterial catheter in infantile aortic aneurysm is strongly circumstantial but essentially speculative. While there is a reasonable suspicion that the infection is introduced via the catheter itself, there is no way of disproving that such catheters do not become secondarily colonised from sepsis originating elsewhere, the most likely source in ventilated preterm infants being the lungs. The increase in the number of reports of this rare condition in recent years probably reflects the greater use of sophisticated technology for the care of the preterm infant. Although still apparently a rare complication of arterial catheterisation and sepsis there is no way of knowing how many infants are surviving and who may yet present with symptoms referable to their aortic aneurysm in later childhood.

We thank Dr M L Chiswick for access to the case records of this patient.

\section{References}

1 Mitchell R G, Claireaux A E. Mycotic aneurysm of the abdominal aorta. Arch Dis Child 1952; 27 : 147-52.

2 Rajs J, Finnström $O$, Wesstrom G. Aortic aneurysm developing after umbilical artery catheterisation. Acta Paediatr Scand 1976; 65: 495-8.

3 Malloy $M$ H, Nichols $M$ M. False abdominal aortic aneurysm: an unusual complication of umbilical arterial catheterization for exchange transfusion. J Pediatr 1977; 90: $285-6$.

4 Spangler J G, Kleinberg F, Fulton R E, Barnhorst D A, Ritter D G. False aneurysm of the descending aorta. A complication of umbilical artery catheterization. $\mathrm{Am} \mathrm{J}$ Dis Child 1977; 131: 1258-9.

- Marchal C, Couronne M, Rouquier F, Lapierre H, Greff $\mathbf{M}$, Mulot $\mathrm{O}$. Anévrisme aortique, septicémie à staphylocoque et cathétérisme artériel ombilical chez 2 jumelles prématurées. Arch Fr Pediatr 1978; 35: 74-81.

- Howorth M B, Jr. Aneurysm of abdominal aorta in the newborn infant. $N$ Engl J Med 1967; 276: 1133-4.

7 Barson A J. Fatal pseudomonas aeruginosa bronchopneumonia in a children's hospital. Arch Dis Child 1971; 46: 55-60.

- Viart P, Cattelain C, Gallez A. Acquired pulmonary artery aneurysm in an infant. Pediatrics $1980 ; 65$ : 89-93.

Correspondence to Dr A J Barson, Department of Pathology, St Mary's Hospital, Whitworth Park, Manchester M13 0JH.

Received 30 March 1981 